\title{
Correlações entre parâmetros de adaptabilidade e estabilidade da produtividade de óleo em soja
}

\author{
Correlations among oil yield adaptability and stability parameters in soybean

\begin{abstract}
Maurisrael de Moura Rocha* Natal Antonio Vello² Ângela Celis de Almeida Lopes ${ }^{3}$ Sandra Helena Unêda-Trevisoli ${ }^{4}$ Maria Clideana Cabral Maia ${ }^{1}$
\end{abstract}

\section{RESUMO}

O objetivo deste trabalho foi determinar o grau de correlação entre parâmetros que avaliam a adaptabilidade e a estabilidade da produtividade de óleo em soja. Os parâmetros foram estimados a partir da análise de dados resultantes da avaliação de 93 linhagens de soja, divididas em três ciclos de maturação (28 precoces, 38 semiprecoces e 27 intermediárias). Os ensaios foram conduzidos em doze ambientes do município de Piracicaba, SP, Brasil. Os seguintes parâmetros foram correlacionados: média geral $\left(m_{i}\right)$, coeficiente de regressão $\left(b_{i}\right)$, ecovalência de Wricke $\left(\omega_{\mathrm{wi}}\right)$, ecovalência AMMI $\left(\omega_{\mathrm{Ai}}\right)$, variância dos desvios da regressão $\left(\sigma^{2}{ }_{\mathrm{di}}\right)$ e coeficiente de determinação $\left(R^{2}\right)$. Utilizou-se a correlação $(r)$ de Sperman do ordenamento das linhagens, segundo cada parâmetro. As correlações entre mi e bi e entre os parâmetros de estabilidade entre si $\left(\omega_{\mathrm{Wi}}, \omega_{\mathrm{Ai}}, \sigma_{\mathrm{di}}^{2}\right.$ e $\left.R^{2}\right)$ foram positivas $e$ significativas $(0,53<r<0,93)$. As correlações entre os parâmetros de adaptabilidade e estabilidade foram baixas ou negativas $e$ não significativas $(-0,39<r<0,38)$, exceto entre $b_{i}$ e $R_{i}^{2}(r=$ 0,49 e 0,89 para as linhagens precoces e intermediárias, respectivamente). Os parâmetros de estabilidade classificam a ordem das linhagens de forma similar e, provavelmente, pertencem ao mesmo conceito de estabilidade. Os resultados indicam que é difícil a seleção simultânea para adaptabilidade e estabilidade da produtividade de óleo em soja.

Palavras-chave: Glycine max, medidas de associação, interação genótipos $x$ ambientes, previsibilidade.

\section{ABSTRACT}

The objective of this work was to determine the degree of correlation among parameters that evaluate oil yield adaptability and stability in soybean. The parameters were estimated starting from data analysis resultants of the evaluation of 93 soybean lines divided in three maturation cycles (28 early, 38 semiearly and 27 intermediate). The trials were carried out in twelve environments of the municipal district of Piracicaba, São Paulo, Brazil. The following parameters were correlated: grand mean $\left(m_{i}\right)$, regression coefficient $\left(b_{i}\right)$, Wricke ecovalence $\left(\omega_{\mathrm{di}}\right)$, AMMI ecovalence $\left(\omega_{\mathrm{Ai}}\right)$, regression deviations variance $\left(\sigma^{2}{ }_{\mathrm{di}}\right)$ and determination coefficient $\left(R^{2}\right)$. The parameters were compared using the ranking Sperman correlation of the lines, according to each parameter. The correlations among $m_{i}$ and $b_{i}$ and among the parameters of stability to each other $\left(\omega_{\mathrm{di}}, \omega_{\mathrm{Ai}}, \sigma^{2}{ }_{\mathrm{di}}\right.$ and $\left.R^{2}\right)$ were positive and significant $(0.53<r<0,93)$. The correlations between the adaptability and stability parameters were low or negatives and not significant $(-0.39<r<0.38)$, except among $b_{i}$ and $R^{2}{ }_{i}$ ( $r=0.49$ and 0.89 , for the early and intermediate lines, respectively). The stability parameters present rank orders of the lines in a similar way and, probably, they belong to the same stabillity concept. The results indicated that is difficult the simultaneous selection for oil yield adaptability and stability in soybean.

Key words: Glycine max, association measures, genotype by environment interaction, predictability.

\section{INTRODUÇÃO}

No Brasil, a soja é cultivada em considerável diversidade de ambientes, desde as altas latitudes (Sudeste e Sul) até baixas latitudes (Centro-Oeste,

${ }^{1}$ Embrapa Meio-Norte, CP 01, 64006-220, Teresina, PI, Brasil. E-mail: mccmaia@cpamn.embrapa.br, mmrocha@cpamn.embrapa.br. *Autor para correspondência.

${ }^{2}$ Escola Superior de Agricultura "Luiz de Queiroz" (ESALQ), Universidade de São Paulo (USP), Departamento de Genética, CP 83, 13400-970, Piracicaba, SP, Brasil. E-mail: naavello@esalq.usp.br.

${ }^{3}$ Universidade Federal do Piauí (UFPI), Departamento de Biologia/Centro de Ciências da Natureza, Campus Universitário da Ininga, 64049-550 Teresina, PI, Brasil. E-mail: acalopes@ufpi.br.

${ }^{4}$ Faculdade de Ciências Agrárias e Veterinárias de Jaboticabal, Universidade Estadual Paulista (UNESP), Departamento de Produção Vegetal, Via de acesso Prof. Paulo Donato Castellane, S/N, 14884-900, Jaboticabal, SP. E-mail: shutrevi@fcav.unesp.br. 
Nordeste e Norte). A seleção de genótipos com alta produtividade de grãos e elevados teores de proteína e óleo é o principal objetivo dos programas de melhoramento (ROCHA \& VELLO, 1999). A interação genótipos $\mathrm{x}$ ambientes (GxE) constitui-se num dos maiores problemas dos programas de melhoramento de qualquer espécie, seja na fase de seleção ou na de recomendação de cultivares. Entre as alternativas para se amenizar a influência dessa interação, tem sido recomendado o emprego de cultivares com ampla adaptabilidade e boa estabilidade. A adaptabilidade é a capacidade de os genótipos aproveitarem vantajosamente o estímulo do ambiente; a estabilidade é a capacidade de os genótipos mostrarem um comportamento altamente previsível em função do estímulo do ambiente (CRUZ \& CARNEIRO, 2003).

As metodologias que analisam a estabilidade são baseadas no princípio da existência de interação GxE. No entanto, diferem nos conceitos de estabilidade e nos procedimentos estatísticos adotados (ROCHA et al., 2004). Os procedimentos para análise biométrica da estabilidade podem ser baseados na variância da interação GxE, na regressão linear simples e múltipla, na regressão quadrática, nos modelos não lineares e não paramétricos, nos métodos multivariados e nos métodos que integram a análise comum de variância (método univariado) com a análise de componentes principais (método multivariado).

Uma das formas utilizadas para comparar metodologias paramétricas que avaliam a adaptabilidade e a estabilidade tem sido a análise da correlação entre seus parâmetros. Nesse sentido, as correlações mais usadas são as de Sperman e Pearson. Essa abordagem tem como principal objetivo verificar similaridades ou divergências entre os parâmetros quanto ao ordenamento dos genótipos nos diversos ambientes.

Um estudo comparando diferentes parâmetros de adaptabilidade e estabilidade foi realizado por DASHIELL et al. (1994), os quais compararam a média geral $\left(\mathrm{m}_{\mathrm{i}}\right)$, o coeficiente de regressão $\left(\mathrm{b}_{\mathrm{i}}\right)$ e a variância dos desvios da regressão $\left(\sigma_{\mathrm{di}}^{2}\right)$ de EBERHART \& RUSSEL (1966). Am foi correlacionada positivamente com o $b_{i}(r=0,50)$ e a $\sigma_{d i}^{2}(r=0,03)$. No entanto, esta última correlação foi quase nula e não significativa e o $b_{i}$ foi negativamente correlacionado com a $\sigma^{2}$ $(r=-0,11)$. Em um outro trabalho conduzido por YUE et al. (1997), constatou-se que o $b_{i}$ foi negativamente correlacionado com a $\omega_{\mathrm{di}}$ e a $\sigma_{\mathrm{di}}^{2}$, ficando estes, significativamente e positivamente correlacionados entre si. Correlações baixas entre a $\omega_{\mathrm{di}}$ e a $\mathrm{m}_{\mathrm{i}}$ foram obtidas por MIRANDA (1999), e entre o b $b_{i}$ e a $\sigma^{2}{ }_{\text {di }}$ por MAURO et al. (2000). O objetivo deste trabalho foi determinar o grau de correlação entre parâmetros de adaptabilidade e estabilidade da produtividade de óleo em soja.

\section{MATERIAL E MÉTODOS}

O caráter produtividade de óleo foi avaliado em 93 linhagens de soja pertencentes a três ciclos de maturação: precoce (28 linhagens), semiprecoce (38 linhagens) e intermediário (27 linhagens). As linhagens foram avaliadas em três locais do município de Piracicaba, SP (Anhembi, Areão e ESALQ), durante quatro anos agrícolas (1996/97, 1997/98, 1998/99 e 1999/ 00), totalizando 12 ambientes. Os experimentos foram conduzidos separadamente para cada ciclo de maturação em delineamento de blocos incompletos estratificados em grupos de tratamentos (regulares: linhagens e não regulares: testemunhas), com duas repetições. O caráter produtividade de óleo foi estimado por meio da fórmula $\mathrm{PO}=(\mathrm{PG} \times \mathrm{OL}) / 100$, em que $\mathrm{PO}$ é a produtividade de óleo em $\mathrm{kg} \mathrm{ha}^{-1}$, $\mathrm{PG}$ é a produtividade de grãos em kg ha-1 e OL é a porcentagem de óleo. Esta última foi obtida por meio de leitura em Espectrômetro de Ressonância Nuclear Magnética a partir de amostras de sementes de cada linhagem, segundo ROCHA (2002).

A média geral de cada genótipo foi estimada de acordo com a equação $m_{i}=Y_{i} / e$, em que $Y_{i j}$ é a produtividade de óleo do i-ésimo genótipo em todos os ambientes; e "e", o número de ambientes. A ecovalência de Wricke $\left(\omega_{\mathrm{di}}\right)$, parâmetro de estabilidade proposto por WRICKE (1965), foi estimada por meio

da equação $\omega_{\mathrm{w}_{\mathrm{i}}}=\sum_{\mathrm{j}=1}^{\mathrm{n}}(\text { ge })_{\mathrm{ij}}^{2}$, com

$(g e)_{i j}=Y_{i j}-Y_{i}-Y_{j}-Y$,em que $Y_{i j}$ é a média do genótipo "i" no ambiente "j"; $Y_{i}$ é a média do genótipo "i" em todos os ambientes; $Y_{j}$ é a média do ambiente "j" para todos os genótipos e $Y=\mathrm{m}_{\mathrm{i}}$ é a média geral. O coeficiente de regressão $\left(b_{i}\right)$, parâmetro de adaptabilidade proposto por EBERHART \& RUSSEL (1966), foi estimado de acordo com a equação $b_{i}=\sum_{j=1}^{n} Y_{i j} I_{j} / \sum_{j=1}^{n} I_{j}^{2}$ , em que $Y_{i j}$ é a média do genótipo "i”" no ambiente “j”; $I_{j}$ é o índice ambiental, estimado por meio da equação

$$
I j=\left[\left(Y_{j} / g\right)-(Y / g e)\right]
$$

tal que $\quad \sum^{\mathrm{n}} \mathrm{I}_{\mathrm{j}}=0$, em que $Y_{j}$ é a média de todos os genótipos ñ no ambiente “ $\mathrm{j}$ ”; $Y=\mathrm{m}_{\mathrm{i}}$ é a média geral; $\mathrm{g}$ é o número de genótipos e "e" é o número de ambientes. 
A variância dos desvios da regressão $\left(\sigma_{\mathrm{d}_{\mathrm{i}}}^{2}\right)$, parâmetro de estabilidade proposto por EBERHART \& RUSSEL (1966), foi obtida segundo a equação,

$$
\sigma_{d i}^{2}=\left[\left(Q M D_{i}\right)(Q M R / r)\right]
$$

em que $\mathrm{QMD}_{\mathrm{i}}$ é o quadrado médio dos desvios da regressão do genótipo “i”; QMR é o quadrado médio do resíduo e r é o número de repetições. O coeficiente de determinação $\left(\mathrm{R}_{\mathrm{i}}^{2}\right)$, parâmetro auxiliar de estabilidade, sugerido por PINTHUS (1973), foi obtido de acordo com a equação,

$$
R_{i}^{2}=\left[(S Q R . \text { Line } a r)_{i} / S Q\left(A / G_{i}\right)\right] X 100
$$

em que (SQR.linear) $)_{i}$ é a soma de quadrados da regressão linear do genótipo “i” e $\mathrm{SQ}\left(\mathrm{A} / \mathrm{G}_{\mathrm{i}}\right)$ é a soma de quadrados de ambientes dentro do genótipo “i”. A ecovalência AMMI $\left(\omega_{\mathrm{Ai}}\right)$, parâmetro de estabilidade proposto por ROCHA (2002), foi estimada de acordo com a equação,

$$
\omega_{\mathrm{A}_{\mathrm{i}}}=\sum_{\mathrm{j}=1}^{N}\left(\mathrm{GxE}_{\text {AMMI } k}\right)_{\mathrm{ij}}^{2}
$$

em que $\omega_{A i}$ é a interação predita para o genótipo "i”" pelo modelo AMMIk selecionado; $\mathrm{N}$ é o número de componentes principais retidos no modelo; GxEAMMIk é estimada segundo a equação

$$
\left(G X E_{\text {AMMIK }}\right)_{i j}=Y_{i j}-Y_{i}-Y_{j}-Y
$$

em que $Y_{i j}$ é a média do genótipo “i” no ambiente “j” predita pelo modelo AMMI $k$ selecionado; $Y_{i}$ é a média do genótipo "i” predita pelo modelo AMMIk selecionado; $\mathrm{Y}_{\mathrm{j}}$ é a média do ambiente “j” predita pelo modelo AMMIk selecionado e $\mathrm{Y}=\mathrm{m}_{\mathrm{i}}$ é a média geral predita pelo modelo AMMIk selecionado.

Na metodologia AMMI (GAUCH \& ZOBEL, 1996), a soma de quadrado da interação GxE é decomposta pela Análise de Componentes Principais (ACP) e compreende uma família de modelos: AMMI0 (nenhum eixo da ACP da interação GxE é considerado no modelo, ou seja, há ausência de interação GxE); AMMI1 (apenas o primeiro eixo da ACP da interação GxE é considerado no modelo); AMMI2 (apenas o primeiro e segundo eixos da ACP da interação GxE são considerados no modelo), AMMI3 (apenas o primeiro, segundo e terceiro eixos da ACP da interação GxE são considerados no modelo),..., AMMIk (apenas os k primeiros eixos da ACP da interação GxE são considerados no modelo). Assim, $k$, corresponde ao número de eixos ou componentes principais da interação GxE incorporados pelo modelo selecionado. A seleção do número de eixos a ser incorporado pelo modelo pode ser realizada por um teste paramétrico, no caso, o teste FR proposto por CORNELIUS et al. (1996) e adotado por ROCHA (2002). É com base na interação GxE predita pelo modelo selecioando que a ecovalência $\operatorname{AMMI}\left(\omega_{\mathrm{Ai}}\right)$ é estimada.

A comparação entre parâmetros que avaliam a adaptabilidade e a estabilidade foi realizada através da correlação de Spearman $\left(r_{s}\right)$ entre o ordenamento das linhagens, de acordo com cada um dos parâmetros. Para efeito de ordenamento, adotou-se os seguintes parâmetros: média $\left(\mathrm{m}_{\mathrm{i}}\right)$, coeficiente de regressão de Ebehart e Russel $\left(b_{i}\right)$, ecovalência de Wricke $\left(\omega_{w_{i}}\right)$, ecovalência AMMI $\left(\omega_{\mathrm{Ai}}\right)$, variância dos desvios da regressão $\left(\sigma_{\mathrm{di}}^{2}\right)$ e o coeficiente de determinação $\left(R_{i}^{2}\right)$. O coeficiente de correlação de Spearman (rs) correspondeu à correlação entre o número de ordem de cada linhagem de acordo com os parâmetros de adaptabilidade e estabilidade, estimado conforme a equação apresentada por MIRANDA (1999), descrita por $r_{s} 1-\left[6 \Sigma\left(r_{i j}-r_{i j}\right)^{2} / g\left(g^{2}-1\right)\right]$ em que $r_{s}$ é o coeficiente de correlação de Spearman; $r_{i j}$ é a ordem de classificação da linhagem i pelo parâmetro j; $r_{i j}$, é a ordem de classificação do genótipo i pelo parầmetro j' e g é o número de pares $\left(\mathrm{r}_{\mathrm{ij}}, \mathrm{r}_{\mathrm{ij}}\right)$, que é igual ao número de genótipos.

\section{RESULTADOS E DISCUSSÃO}

Os resultados das estimativas dos coeficientes de correlação de Spearman aplicados às ordens crescentes para os parâmetros de estabilidade $\left(\omega_{\mathrm{Wi}}, \omega_{\mathrm{Ai}}, \sigma^{2}{ }_{\mathrm{di}}\right.$ e $\left.R^{2}{ }_{i}\right)$ e às ordens decrescentes para os parâmetros de adaptabilidade $\left(\mathrm{m}_{\mathrm{i}} \mathrm{e} \mathrm{b}_{\mathrm{i}}\right)$, para cada par de parâmetros, mostraram que $45 \%$ das correlações estimadas apresentou significância $(\mathrm{P}<0,01)$, evidenciando um grau intermediário de associação no conjunto dos parâmetros considerados (Tabela 1).

Analisando as correlações entre os diversos parâmetros e a média geral da produtividade de óleo $\left(\mathrm{m}_{\mathrm{i}}\right)$, observou-se que a mais alta correlação ocorreu com o $b_{i}$ (Tabela 1). As estimativas foram maiores e significativas para as linhagens de ciclo intermediário $(\mathrm{r}=0,66)$ e menores e não significativas para as linhagens de ciclo precoce $(r=0,22)$ e semiprecoce $(r=0,31)$. Essa tendência positiva de associação entre a $m_{i}$ e o $b_{i}$ era esperada, uma vez que o bi eleva-se à medida que a linhagem possui valores médios de produtividade de óleo maiores na maioria dos ambientes. A mi apresentou baixa associação com os parâmetros de estabilidade $\left(\omega_{\mathrm{Wi}}, \omega_{\mathrm{Ai}}, \sigma^{2}{ }_{\mathrm{di}}\right.$ e $\left.R^{2}{ }_{i}\right)$ em todos os ciclos de maturação. Esses resultados concordam com aqueles obtidos em estudos desta natureza com feijão comum (DUARTE \& ZIMMERMANN, 1995; MEKBIB, 2003). Em soja, DASHIELL et al. (1994) encontraram resultados semelhantes para a correlação 
Tabela 1 - Coeficientes de correlação de Spearman entre a média geral $\left(\mathrm{m}_{\mathrm{i}}\right)$, o coeficiente de regressão $\left(\mathrm{b}_{\mathrm{i}}\right)$, a ecovalência de Wricke $\left(\omega_{\mathrm{W}_{\mathrm{i}}}\right)$, a ecovalência AMMI $\left(\omega_{\mathrm{A}_{\mathrm{i}}}\right)$, a variância dos desvios da regressão $\left(\sigma_{d_{i}}^{2}\right)$ e o coeficiente de determinação $\left(\mathrm{R}_{\mathrm{i}}^{2}\right)$ para o caráter produtividade de óleo, obtidos em linhagens de soja pertencentes a três ciclos de maturação. Soja, Piracicaba, SP, 2002.

\begin{tabular}{|c|c|c|c|c|c|}
\hline \multirow[b]{2}{*}{ Parâmetro } & \multicolumn{5}{|c|}{ Linhagens de ciclo precoce } \\
\hline & $b_{i}$ & $\omega_{\mathrm{W}_{\mathrm{i}}}$ & $\omega_{\mathrm{A}_{\mathrm{i}}}(\mathrm{AMMI} 4)^{1}$ & $\sigma_{\mathrm{d}_{\mathrm{i}}}^{2}$ & $\mathrm{R}_{\mathrm{i}}^{2}$ \\
\hline $\mathrm{m}_{\mathrm{i}}$ & $0,22^{\mathrm{ns}}$ & $0,17^{\mathrm{ns}}$ & $0,24^{\mathrm{ns}}$ & $0,06^{\mathrm{ns}}$ & $0,24^{\mathrm{ns}}$ \\
\hline$b_{i}$ & - & $-0,13^{\text {ns }}$ & $-0,11^{\mathrm{ns}}$ & $-0,19^{\text {ns }}$ & $0,49 * *$ \\
\hline$\omega_{\mathrm{W}_{\mathrm{i}}}$ & & - & $0,89^{* *}$ & $0,92 * *$ & $0,75^{* *}$ \\
\hline$\omega_{\mathrm{A}_{\mathrm{i}}}(\mathrm{AMMI} 4)$ & & & - & $0,88 * *$ & $0,69 * *$ \\
\hline \multirow[t]{3}{*}{$\sigma_{\mathrm{d}_{\mathrm{i}}}^{2}$} & & & & - & $0,66^{* *}$ \\
\hline & \multicolumn{5}{|c|}{ Linhagens de ciclo semiprecoce } \\
\hline & $b_{i}$ & $\omega_{\mathrm{W}_{\mathrm{i}}}$ & $\omega_{\mathrm{A}_{\mathrm{i}}}(\mathrm{AMMI} 4)^{1}$ & $\sigma_{\mathrm{d}_{\mathrm{i}}}^{2}$ & $\mathrm{R}_{\mathrm{i}}^{2}$ \\
\hline $\mathrm{m}_{\mathrm{i}}$ & $0,31^{\mathrm{ns}}$ & $0,06^{\mathrm{ns}}$ & $-0,01^{\mathrm{ns}}$ & $0,11^{\mathrm{ns}}$ & $0,19^{\text {ns }}$ \\
\hline$b_{i}$ & - & $-0,31^{\text {ns }}$ & $-0,39^{\text {ns }}$ & $-0,25^{\mathrm{ns}}$ & $0,19^{\mathrm{ns}}$ \\
\hline$\omega_{\mathrm{W}_{\mathrm{i}}}$ & & - & $0,91^{* *}$ & $0,93 * *$ & $0,83^{* *}$ \\
\hline$\omega_{\mathrm{A}_{\mathrm{i}}}(\mathrm{AMMI} 4)$ & & & - & $0,86^{* *}$ & $0,67 * *$ \\
\hline \multirow[t]{3}{*}{$\sigma_{d_{i}}^{2}$} & & & & - & $0,75^{* *}$ \\
\hline & \multicolumn{5}{|c|}{ Linhagens de ciclo intermediário } \\
\hline & $b_{i}$ & $\omega_{\mathrm{W}_{\mathrm{i}}}$ & $\omega_{\mathrm{A}_{\mathrm{i}}}(\mathrm{AMMI} 2)^{2}$ & $\sigma_{\mathrm{d}_{\mathrm{i}}}^{2}$ & $\mathrm{R}_{\mathrm{i}}^{2}$ \\
\hline $\mathrm{m}_{\mathrm{i}}$ & $0,66^{* *}$ & $0,15^{\mathrm{ns}}$ & $0,05^{\mathrm{ns}}$ & $0,15^{\mathrm{ns}}$ & $0,38^{\mathrm{ns}}$ \\
\hline$b_{i}$ & - & $0,16^{\mathrm{ns}}$ & $0,36^{\mathrm{ns}}$ & $0,27^{\mathrm{ns}}$ & $0,89 * *$ \\
\hline$\omega_{\mathrm{W}_{\mathrm{i}}}$ & & - & $0,59 * *$ & $0,89^{* *}$ & $0,54 * *$ \\
\hline$\omega_{\mathrm{A}_{\mathrm{i}}}(\mathrm{AMMI} 2)$ & & & - & $0,36^{\mathrm{ns}}$ & $0,53 * *$ \\
\hline$\sigma_{d_{i}}^{2}$ & & & & - & $0,62 * *$ \\
\hline
\end{tabular}

**Significativo ao nível de $1 \%$ de probabilidade pelo teste $t$.

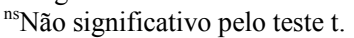

${ }^{1}$ Parâmetro estimado a partir das interações GxE preditas pelo modelo AMMI4, previamente selecionado pelo teste $\mathrm{F}_{\mathrm{R}}(\mathrm{CORNELIUS} \mathrm{et} \mathrm{al.,}$ 1996).

${ }^{2}$ Parâmetro estimado a partir das interações GxE preditas pelo modelo AMMI2, previamente selecionado pelo teste $\mathrm{F}_{\mathrm{R}}$ (CORNELIUS et al., 1996). 
entre a m e os parâmetros $b_{i}(r=0,50)$ e $\sigma_{d i}^{2}(r=0,03)$ e MIRANDA (1999) também obteve baixa correlação entre a $m_{i}$ e a $\omega_{w_{i}}(r=0,02)$.

Entre as estimativas de correlação do $b_{i}$ e os parâmetros de estabilidade $\left(\omega_{\mathrm{Wi}}, \omega_{\mathrm{Ai}}, \sigma_{\mathrm{di}}^{2}\right.$ e $\left.R_{i}^{2}\right)$, destacaram-se como positivas e significativas aquelas com o nas linhagens de ciclo precoce e intermediário $(\mathrm{r}=0,49$ e 0,89 , respectivamente) (Tabela 1). Associações positivas (linhagens de ciclo intermediário) e negativas (linhagens de ciclo precoce e semiprecoce), porém não significativas, foram observadas entre o $b_{\mathrm{i}}$ e os parâmetros $\omega_{\mathrm{Wi}}, \omega_{\mathrm{Ai}} e \sigma_{\mathrm{di}}^{2}$. Isto mostra que o parâmetro

$R_{i}^{2}$ não é adequado para selecionar para estabilidade, já que este é linearmente associado ao parâmetro $b_{i}$. Essa associação é evidente principalmente nas linhagens de ciclo precoce e intermediário, evidenciando que, entre o $R_{i}^{2}$ e a $\sigma_{\text {di }}^{2}$, este último é mais adequado como parâmetro de medida de estabilidade.

Correlações altas, positivas e significativas foram observadas entre os parâmetros de estabilidade $\left(\omega_{\mathrm{Wi}}, \omega_{\mathrm{Ai}}, \sigma_{\mathrm{di}}^{2}\right.$ e $\left.R_{i}^{2}\right)$, para a maioria das estimativas e ciclos de maturação (Tabela 1 ). $\mathrm{A} \omega_{\mathrm{Wi}}$ apresentou maior associação com a $\sigma_{\mathrm{di}}^{2}(\mathrm{r}=0,89$ a 0,93$)$, sugerindo que esses dois parâmetros são fortemente correlacionados. Resultado semelhante foi obtido por MEKBIB (2003) em feijão comum, que também obteve alta correlação entre $\omega_{\mathrm{Wi}}$ e $\sigma_{d i}^{2}(r=0,92)$. Como a magnitude da ecovalência depende principalmente da magnitude do quadrado médio dos desvios, espera-se que a $\omega_{\mathrm{wi}} \mathrm{e}$ sejam altamente correlacionados. Portanto, recomendase o uso de um ou outro parâmetro como medida da estabilidade agronômica. Como a $\sigma_{\mathrm{di}}^{2}$ é um parâmetro associado aos desvios da regressão, garante maiores informações a respeito do comportamento individual de cada genótipo e é preferível em relação ao parâmetro $\omega_{\mathrm{Wi}}$, por ser este menos informativo.

Correlações entre a $\omega_{\mathrm{Wi}}$ e o $R_{i}^{2}$ geralmente foram altas em todos os ciclos de maturação $(r=0,54$ a 0,89$)$; já entre a $\omega_{\mathrm{Wi}}$ e a $\omega_{\mathrm{Ai}}$, as correlações variaram de 0,59 a 0,91 (Tabela 1). Associações altas e significativas também foram observadas entre a $\sigma_{\text {di }}^{2} \mathrm{e}$ o $R_{i}^{2}(\mathrm{r}=0,54$ a 0,83$)$ e entre a $\sigma^{2}{ }_{\mathrm{di}}$ e a $\omega_{\mathrm{Ai}}(\mathrm{r}=0,56 \mathrm{a}$ $0,88)$. O $R_{i}^{2}$ apresentou estimativas positivas e significativas de $r>0,50$ com a $\quad \omega_{\mathrm{Ai}}$, apresentando uma média de $r=0,60$. Esses resultados discordam daqueles obtidos por MEKBIB (2003), que obteve correlações altas e significativas, porém negativas, entre $R_{i}^{2}$ e os parâmetros $\sigma_{\mathrm{di}}^{2}$ e $\omega_{\mathrm{Wi}}$.

Observou-se que as correlações entre a $\omega_{\mathrm{Ai}}$ com a $\omega_{\mathrm{Wi}}$ e a $\sigma_{\text {di }}^{2}$, no geral, foram positivas, altas, significativas, e, de certa forma, similares. Nos casos em que a estimativa foi obtida a partir do modelo AMMI com maior número de eixos da ACP (AMMI4), as correlações tenderam a serem mais altas. Uma explicação, no caso da $\omega_{\mathrm{Ai}}$ (AMMI4) e a $\omega_{\mathrm{Wi}}$, é que as estimativas preditas pela ecovalência AMMI4 são mais próximas daquelas estimadas pela ecovalência de Wricke $\left(\omega_{\mathrm{wi}}\right)$. Ambas foram estimadas com base na soma de quadrados da interação (SQGxE). No entanto, no cálculo da $\omega_{\mathrm{Wi}}$ é considerada a SQGxE total, enquanto que, no cálculo da $\omega_{\mathrm{Ai}}$, considera-se apenas parte da SQGxE; parte essa que contém a maior porção do padrão (variação agromicamente importante da interação $\mathrm{GxE}$ ) e que é selecionada por meio de um teste, no caso, do teste FR (CORNELIUS et al., 1996).

$\mathrm{Na}$ análise AMMI, o padrão apresenta-se em maior concentração nos primeiros componentes principais (por ex.: AMMI1 e AMMI2) e em menor concentração nos eixos remanescentes (ROCHA, 2002). Estes são descartados por serem pobres em padrão e ricos em ruídos (variação aleatória e sem importância agronômica). Assim, modelos AMMI que englobam muitos eixos da ACP (por ex.: AMMI4) tendem a se aproximar do modelo completo (SQGXE total), que é a base de cálculo da $\omega_{\mathrm{Ai}}$.

Por meio da metodologia AMMI, a SQGxE total foi decomposta em onze eixos pela ACP, e o teste FR selecionou os dois primeiros eixos (modelo AMMI2) para a interação GxE das linhagens de ciclo intermediário e os quatro primeiros eixos (modelo AMMI4) para a interação $\mathrm{GxE}$ das linhagens de ciclo precoce e semipreccoe. Maiores detalhes sobre essa decomposição podem ser obtidos no trabalho de ROCHA(2002).

No caso dos parâmetros $\omega_{\mathrm{Ai}}$ e $\sigma^{2}{ }_{\mathrm{di}}$, a explicação acima é válida em parte, já que a obtenção da $\sigma_{\text {di }}^{2}$ também envolve a SQGxE, mas também engloba a soma de quadrados de ambientes (SQE). Nota-se que não houve uma tendência em termos de magnitude para as correlações entre a $\omega_{\mathrm{Ai}}$ com a $\omega_{\mathrm{Wi}}$ ou com a $\sigma_{\mathrm{di}}^{2}$. Ora a correlação foi maior entre a $\omega_{\mathrm{Ai}}$ e a $\omega_{\mathrm{Wi}}$, ora foi maior entre a $\omega_{\mathrm{Ai}}$ e a $\sigma_{\text {di }}^{2}$.

$\mathrm{O}$ ordenamento dos genótipos em cada ambiente estimado por modelos AMMI que englobam poucos eixos da ACP da interação GxE, por exemplo, AMMI1, foi diferente do ordenhamento que englobava todos os eixos (modelo completo) em um trabalho conduzido por GAUCH \& ZOBEL (1996). Esses autores, trabalhando com espécies diferentes (trigo, soja e milho), encontraram que a porcentagem para diferenças no ordenamento dos genótipos foi sempre maior em modelos reduzidos (por ex.: AMMI1 e AMMI2), quando comparados com o ordenamento realizado com médias não ajustadas ou modelo completo (AMMI11), 
estimadas com base na SQGxE total. Observou-se também essa tendência, já que, nos casos em que os modelos eram mais reduzidos, as correlações entre a $\omega_{\mathrm{Ai}}$ e os parâmetros estimados com base na SQGxE total $\left(\omega_{\mathrm{Wi}}\right.$ e $\left.\sigma_{\mathrm{di}}^{2}\right)$ foram sempre mais baixas, como mostram as estimativas entre a $\omega_{\mathrm{Ai}}$ com a $\sigma_{\mathrm{di}}^{2}$ e a $\omega_{\mathrm{W}}$ (Tabela 1).

O uso de parâmetros pertencentes a diferentes conceitos pode levar a diferentes ordenamentos de genótipos em termos de sua estabilidade (LIN et al., 1986). Os resultados de correlações observadas entre os parâmetros $\omega_{\mathrm{Ai}}, \omega_{\mathrm{Wi}}$ e $\sigma^{2}{ }_{\mathrm{di}}$ indicaram que estes ordenam as linhagens de forma similar, evidenciando que pertencem a um mesmo tipo de conceito em termos de estabilidade. A $\omega_{W_{i}}$ apresenta estabilidade tipo 3 (alta confiabilidade de resposta estimada, segundo LIN et al., 1986). A estabilidade avaliada pela ecovalência de $\operatorname{Wricke}\left(\sigma_{\mathrm{di}}^{2}\right)$, não somente reflete a estabilidade tipo 2, mas também reflete o conceito de estabilidade tipo 3. Em relação a $\omega_{\mathrm{Ai}}$, por ser estimada da mesma forma que a $\omega_{\mathrm{Ai}}, \omega_{\mathrm{Wi}}$ também pode refletir a estabilidade tipo 3 , diferindo apenas na vantagem adicional de este parâmetro avaliar melhor o padrão subjacente à interação $\mathrm{GxE}$, segundo DUARTE \& ZIMMERMANN (1995).

A análise de correlação mostrou que os parâmetros que avaliam a estabilidade $\left(\omega_{\mathrm{Ai}}, \omega_{\mathrm{Wi}} \mathrm{e}\right.$ $\left.\sigma^{2}{ }_{\mathrm{di}}\right)$, nos três ciclos de maturação, ordenaram as linhagens de forma similar, com pequenas mudanças no ordenamento de poucas linhagens, mudanças determinadas, principalmente pelo parâmetro $\omega_{\mathrm{Ai}}$. Isso porque este parâmetro apresentou magnitudes menores para os coeficientes de correlação com a $\omega_{\mathrm{Wi}}$ e a $\sigma_{\text {di }}^{2}$ em comparação com aqueles obtidos entre a $\omega_{\mathrm{wi}}$ e a $\sigma^{2}{ }_{\mathrm{di}}$. Estas correlações não foram perfeitas $(\mathrm{r}=1)$, mas foram altas, positivas e significativas, sugerindo que estes parâmetros pertencem a um mesmo conceito de estabilidade, provavelmente o conceito tipo 3, preconizado por LIN et al. (1986).

\section{CONCLUSÃO}

A seleção simultânea para adaptabilidade e estabilidade da produtividade de óleo em soja é difícil e requer a associação entre um parâmetro de estabilidade e outro de adaptabilidade.

\section{AGRADECIMENTOS}

À Coordenação de Aperfeiçoamento de Pessoal de Nível Superior (Capes) e ao Conselho Nacional de Desenvolvimento Científico e Tecnológico ( $\mathrm{CNPq})$, pelo apoio financeiro às diferentes fases desta pesquisa.

Ao Dr. João Batista Duarte, pelas sugestões na estimação da ecovalência AMMI.
Aos funcionários A. R. Cogo, A. Didoné e M. C. Nekatschalow, pelo auxílio na condução dos experimentos.

\section{REFERÊNCIAS}

CORNELIUS, P.L. et al. Statistical tests and stimators of multiplicative models for genotype-by-environment interaction. In: KANG, MS.; GAUCH, H.G. (Ed). Genotypeby-environment interaction. New York: CRC, 1996. p.199234.

CRUZ, C.D.; CARNEIRO, P.C.S. Modelos biométricos aplicados ao melhoramento genético. Viçosa: UFV, 2003. V.2, 585p.

DASHIEL, K.E. et al. Genotype x environment interaction and simultaneous selection for high yield and stability in soybeans (Glycine max (L.) Merrill). Annals of Applied Biology, v.124, n.1, p.133-139, 1994

DUARTE, J.B.; ZIMMERMANN, M.J.O. Correlation among yield stability parameters in common bean. Crop Science, v.35, n.3, p.905-912, 1995 .

EBERHART, S.A.; RUSSEL, W.A. Stability parameters for comparing varieties. Crop Science, v.6, n.1, p.36-40, 1966.

GAUCH, H.G.; ZOBEL, R.W. AMMI analysis of yield trials. In: KANG, MS.; GAUCH, H.G. (Ed). Genotype-by-environment interaction. New York: CRC, 1996. p.85-122.

LIN, C.S. et al. Stability analysis: where do we stand? Crop Science, v.26, n.5, p.894-900, 1986.

MAURO, A.O. et al. Correlação entre medidas paramétricas e não paramétricas de estabilidade em soja. Pesquisa Agropecuária Brasileira, v.35, n.4, p.687-696, 2000

MIRANDA, F.T.S. Interação genótipos $\mathbf{x}$ ambientes em linhagens de soja selecionadas para resistência ao nematóide de cisto. 1999. 141f. Dissertação (Mestrado em Genética e Melhoramento de Plantas) - Curso de Pós-graduação em Agronomia, Escola Superior de Agricultura "Luiz de Queiroz", Universidade de São Paulo.

MEKBIB, F. Yield stability in common bean (Phaseolus vulgaris L.) genotypes. Euphytica, v. 130, p. 147-153, 2003.

PINTHUS, M.J. Estimate of genotypic value: a proposed method. Euphytica, v.22, n.1, p.121-123, 1973.

ROCHA, M.M. Seleção de linhagens experimentais de soja para adaptabilidade e estabilidade fenotípica. 2002. 173f. Tese (Doutorado em Genética e Melhoramento de Plantas) - Curso de Pós-graduação em Agronomia, Escola Superior de Agricultura "Luiz de Queiroz", Universidade de São Paulo.

ROCHA, M.M.; VELLO, N.A. Interação genótipos e locais para rendimento de grãos de linhagens de soja com diferentes ciclos de maturação. Bragantia, v.58, n.1, p.69-81, 1999.

ROCHA, M.M. et al. Yield stability of soybean lines using additive main effects and multiplicative interaction analysis AMMI. Crop Breeding and Applied Biotechnology, v.4, n.4, p.391-398, 2004

WRICKE, G. Zur berechnung der okovalenz bei sommerweizen und hafer. Z. Pflanzenzuchtg, v.52, p.127-138, 1965

YUE, G.L. et al. Evaluation of soybean cultivars using parametric and nonparametric stability estimates. Plant Breeding, v.116, n.3, p.271-275, 1997

Ciência Rural, v.36, n3, mai-jun, 2006. 